\title{
COMPACTAÇÃO CAUSADA PELO MANEJO DE PLANTAS INVASORAS EM LATOSSOLO VERMELHO-AMARELO CULTIVADO COM CAFEEIROS ${ }^{(1)}$
}

\author{
Paula Sant'Anna Moreira Pais ${ }^{(2)}$, Moacir de Souza Dias Junior ${ }^{(3)}$, \\ Gislene Aparecida dos Santos ${ }^{(4)}$, Adriana Cristina Dias ${ }^{(5)}$, Paulo \\ Tácito Gontijo Guimarães ${ }^{(6)}$ \& Elifas Nunes de Alcântara ${ }^{(6)}$
}

\begin{abstract}
RESUMO
O manejo de plantas invasoras é considerado uma das principais atividades que promovem degradação da estrutura do solo em lavouras cafeeiras, devido à compactação do solo causada pelas operações de controle de plantas invasoras. $O$ objetivo deste estudo foi determinar o modelo de capacidade de suporte de carga para o manejo de plantas invasoras sem capina, bem como, utilizando esse modelo, qual manejo de plantas invasoras causa menor ou maior compactação do solo. Este estudo foi realizado em um Latossolo Vermelho-Amarelo (LVA) cultivado com cafeeiros da cutivar Topázio MG 1190 desde 2006, localizado na Fazenda Experimental da EPAMIG, na comunidade Farias, em Lavras-MG. Os manejos de plantas invasoras avaliados foram: na linha de tráfego da entrelinha - grade de discos, herbicida de pós-emergência, herbicida de pré-emergência, roçadora e trincha; e no centro da entrelinha, onde não houve tráfego - amendoim forrageiro (Arachis pintoi), braquiária (Brachiaria decumbens), capina manual, crotalária (Crotalaria juncea) e soja (Glicine max L). A amostragem consistiu de duas etapas: uma para determinar o modelo de capacidade de suporte de carga para o manejo de plantas invasoras sem capina; e outra para avaliar a compactação promovida pelos outros manejos de plantas invasoras. A fim de determinar o modelo de capacidade de suporte de carga para o manejo sem capina, foram coletadas no
\end{abstract}

(1) Parte da Dissertação de Mestrado da primeira autora. Recebido para publicação em 15 de dezembro de 2010 e aprovado em 17 de agosto de 2011.

(2) Engenheira-Agrônoma, Doutoranda em Ciência do Solo no Departamento de Ciência do Solo, Universidade Federal de Lavras - DCS/UFLA. Caixa Postal 3037, CEP 37200-000 Lavras (MG). Bolsista Embrapa/Café. E-mail: paulaufla@yahoo.com.br

(3) Engenheiro Agrícola, PhD em Crop and Soil Science. Professor do Departamento de Ciência do Solo, UFLA. Bolsista CNPq e Pesquisador Mineiro da FAPEMIG. Email: msouzadj@dcs.ufla.br

(4) Engenheira Agrícola, Bolsista de Pós-doutorado - PNPD/CAPES - Programa de Pós-graduação em Manejo de Solo e da Água - DSER - CCA/UFPB. CEP 58397-000 Areia (PB). E-mail: gisantosolos@yahoo.com.br

(5) Engenheira-Agrônoma, Mestranda em Ciência do Solo no Departamento de Ciência do Solo, DCS/UFLA. Bolsista CAPES. Email: drikinha.ufla@gmail.com

(6) Pesquisador da Empresa de Pesquisa Agropecuária de Minas Gerais - EPAMIG. Campus UFLA. E-mails: paulotgg@epamig.ufla.br; elifas@epamig.ufla.br 
centro da entrelinha 20 amostras com estrutura indeformada nas profundidades de 0-3, 10-13 e 25-28 cm, totalizando 60 amostras. Essas amostras foram submetidas ao ensaio de compressão uniaxial para obter as pressões de pré-consolidação e as umidades volumétricas, que foram usadas para determinar o modelo de capacidade de suporte de carga. Para determinar a compactação causada pelos manejos de plantas invasoras, realizados por meio do controle mecânico, foram coletadas em janeiro de 2010, nas linhas de tráfego das entrelinhas, 180 amostras com estrutura indeformada (5 manejos x 3 profundidades x 12 amostras de solo com estrutura indeformada); para os manejos de plantas invasoras realizados com o uso de plantas de cobertura, foram coletadas em janeiro de 2010, no centro das entrelinhas, 180 amostras com estrutura indeformada (5 manejos $x 3$ profundidades $x 12$ amostras de solo com estrutura indeformada). Essas amostras foram submetidas ao ensaio de compressão uniaxial, a fim de obter as pressões de pré-consolidação e as umidades volumétricas após a implantação dos manejos de plantas invasoras, e usadas nos critérios propostos por Dias Junior et al. (2005) para determinar a compactação causada por esses manejos. $O$ uso dos modelos de capacidade de suporte de carga e das pressões de pré-consolidação determinadas após a implantação dos manejos de plantas invasoras permitiu identificar os manejos grade de discos, roçadora e trincha como os que promoveram maior compactação; os manejos braquiária, crotalária e soja foram os que causaram menor compactação nas três profundidades estudadas.

Termos de indexação: sustentabilidade, pressão de pré-consolidação, modelagem da capacidade de suporte de carga, Coffea arabica $\mathrm{L}$.

\section{SUMMARY: IMPACTS CAUSED BY DIFFERENT WEED MANAGEMENTS ON A RED-YELLOW LATOSOL UNDER COFFEE}

Weed management is considered one of the activities that degrade the soil structure most in coffee crops, due to the soil compaction caused by weed control operations. The objective of this study was to determine the bearing capacity models for no weed control and to use this model to determine which weed management causes smaller or greater soil compaction. The study was conducted on a Red-Yellow Latosol (LVA), under coffee (variety Topazio MG 1190), on the Experimental Farm of EPAMIG in the community Farias, Lavras-MG (latitude $21^{\circ} 14^{\prime} 43^{\prime \prime} S$, longitude $44^{\circ} 59^{\prime} 59^{\prime \prime} W$ ). The following weed managements were assessed: a) in the traffic line of the inter-rows-disc harrow, post-emergence herbicide, pre-emergence herbicide, mower and brush and b) in the center of the inter-rows - peanut (Arachis pintoi), braquiaria (Brachiaria decumbens), hand weeding, sunhemp (Crotalaria juncea) and soybean (Glycine $\max L$ ). The sampling consisted of two stages, one to determine the bearing capacity models for the treatment no weeding and another to assess the compaction caused by the other weed management. To determine the bearing capacity model for the treatment no weeding, 20 undisturbed soil samples from the layers $0-3,10-13$ and $25-28 \mathrm{~cm}$ were collected in the middle of the inter-rows, totaling 60 samples. These samples were subjected to the uniaxial compression test to obtain the preconsolidation pressure and the volumetric water content, which were used to determine the bearing capacity model. To determine the compaction caused by the other weed managements, based on mechanical control, 180 samples with undisturbed structure were collected in January 2010 from the traffic lines of the inter-rows, (5 weed management $x 3$ depths $x 12$ soil samples with undisturbed structure). For the weed managements using cover crops, in January 2010, 180 samples with undisturbed structure (5 weed management $x 3$ depths $x 12$ soil samples with undisturbed structure) were collected from the middle of the inter-rows These samples were submitted to the uniaxial compression test to determine preconsolidation pressure and the volumetric water content after applying the weed managements and were used in the criteria proposed by Dias Junior et al. (2005) to determine the compaction caused by these managements. By the bearing capacity models and the preconsolidation pressure determined after implementing the weed managements, the treatments disc harrow, mower and brush were detected for causing greatest soil compaction and braquiaria, sunhemp and soybean as causing least compaction in the three studied layers.

Index terms: sustainability, preconsolidation pressure, modeling of the bearing capacity, Coffea arabica $L$. 


\section{INTRODUÇÃO}

O Brasil é o maior produtor e exportador de café e o segundo maior consumidor do produto (ABIC, 2009), detendo cerca de $30 \%$ do mercado mundial de café; a safra estimada para 2011 é de 43,54 milhões de sacas de $60 \mathrm{~kg}$ de café beneficiado. A área plantada atualmente é de 2,06 milhões de hectares, e o setor emprega direta e indiretamente cerca de 8,4 milhões de trabalhadores. Minas Gerais produziu 22,12 milhões de sacas, totalizando $50,6 \%$ da produção brasileira; a região Sul de Minas Gerais produziu 47,0 \% da produção estadual (CONAB, 2011).

Para obtenção dessa produção, o produtor brasileiro é obrigado a adotar inovações tecnológicas a fim de reduzir custos e melhorar a qualidade de seu produto final, embora ainda com custo final elevado (Marino, 2002), o que pode constituir uma desvantagem quando comparado ao custo de produção de outros países que entraram no mercado do café nas últimas décadas.

Um dos fatores que ajudam a encarecer o produto final está relacionado à correta manutenção do cafezal. O cafeeiro, como outras culturas, é muito sensível à infestação e à concorrência de plantas invasoras, que competem por água, luz e nutrientes, podendo ocorrer queda da produção (Santos et al., 2008), a qual pode variar de 40 a 60 \% (Blanco et al., 1978; Miguel et al., 1980; Santos et al., 2008).

O manejo de plantas invasoras na cultura do cafeeiro está relacionado com o uso, em geral, de máquinas agrícolas, que podem causar compactação do solo (Larson et al., 1989; Dias Junior \& Pierce, 1996; Dias Junior, 2000; Santos et al., 2010) e alteração do meio onde o sistema radicular desenvolve (Gysi, 2001), promovendo degradação da estrutura do solo e redução da produção das culturas (Araujo Junior, 2007).

O manejo de plantas invasoras pode ser feito por meio de métodos que usam plantas de cobertura e de métodos mecânicos que envolvem o uso de máquinas. De acordo com Wildner (1992), o manejo de plantas invasoras realizado com plantas de cobertura, além de reduzir a incidência de plantas invasoras, funciona também como cobertura do solo, possibilitando, além do controle da erosão, a redução das perdas de nutrientes, a atenuação das flutuações da temperatura do solo e a minimização dos efeitos da compactação do solo.

As plantas de cobertura apresentam capacidade de crescer em solos com alta resistência mecânica, criando canais ("bioporos") nos quais as raízes da cultura subsequente podem crescer (Ehlers et al., 1983; Silva \& Rosolem, 2001). Além desse efeito físico de facilitar o crescimento radicular em profundidade, esses bioporos apresentam, também, microclima favorável ao crescimento das raízes, uma vez que há mais substrato orgânico oriundo de exsudatos radiculares ou da decomposição das raízes mortas, que, além de complexar $\mathrm{Al}$, reduzem seu efeito tóxico (Canellas et al., 1999).

Já o manejo de plantas invasoras por métodos mecânicos é considerado uma das principais atividades que promovem degradação da estrutura do solo, devido à compactação do solo causada pelas operações de controle. Desse modo, é importante entender como o manejo de plantas invasoras altera a capacidade de suporte de carga dos solos - principalmente quando feito mecanicamente e em condições inadequadas de umidade do solo - para adaptá-lo de forma condizente, visando maior longevidade e maior produtividade da lavoura cafeeira (Santos, 2006; Araujo Junior et al., 2008).

O objetivo deste estudo foi determinar o modelo de capacidade de suporte de carga para o manejo de plantas invasoras sem capina, bem como, usando esse modelo, qual manejo de plantas invasoras causa menor ou maior compactação do solo.

\section{MATERIAL E MÉTODOS}

O estudo foi realizado em uma área da Fazenda Experimental da EPAMIG (Empresa de Pesquisa Agropecuária de Minas Gerais), situada em Lavras, MG, região do Campo das Vertentes, com latitude de $21^{\circ} 14^{\prime} 43^{\prime \prime}$ S e longitude de $44^{\circ} 59^{\prime} 59^{\prime \prime} \mathrm{W}$ de Greenwich e a uma altitude de $919 \mathrm{~m}$.

O clima é do tipo Cwa, conforme a classificação climática de Köppen. A temperatura média anual está em torno de $19,3^{\circ} \mathrm{C}$, tendo, no mês mais quente e no mais frio, temperaturas médias de $22,1 \mathrm{e} 15,8^{\circ} \mathrm{C}$, respectivamente. A precipitação pluvial anual é de $1.530 \mathrm{~mm}$; a evaporação total do ano, de $1.343 \mathrm{~mm}$; e a umidade relativa média anual, de 76 \% (Brasil, 1992).

$\mathrm{O}$ solo da área em estudo foi classificado como Latossolo Vermelho-Amarelo (LVA) textura argilosa (Embrapa 2006). As caracterizações física e química são apresentadas no quadro 1.

O estudo foi realizado em uma lavoura cafeeira (Coffea arabica L.) implantada em 2006, com a cultivar Topázio MG 1190. De acordo com o histórico, a área, antes da instalação do experimento, era formada por pastagem nativa de campo limpo, com presença de alguns arbustos de cerrado. Para instalação do experimento, foi feita uma limpeza na área, utilizando um trator de esteira; posteriormente, foi feita uma gradagem com um trator traçado na profundidade de $30 \mathrm{~cm}$.

O manejo de plantas invasoras sem capina (SC) foi utilizado para determinação do modelo de capacidade de suporte de carga, por não ter ocorrido nenhuma operação de controle de plantas invasoras desde a implantação do experimento. Assim, esse manejo foi usado para comparar as alterações promovidas pelos 
Quadro 1. Caracterização física e química do Latossolo Vermelho-Amarelo (LVA)

\begin{tabular}{|c|c|c|c|c|c|c|c|c|c|c|c|c|}
\hline Profundidade & Argila & Areia & Silte & Dp & $\mathbf{p H}$ & SB & $\mathbf{t}$ & $\mathbf{T}$ & $\mathbf{V}$ & $\mathbf{m}$ & MO & P. rem \\
\hline $\mathrm{cm}$ & 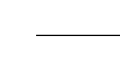 & $-\mathrm{g} \mathrm{kg}^{-1}$ & 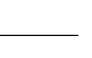 & $\mathrm{Mg} \mathrm{m}^{-3}$ & & $-\mathrm{cm}$ & $l_{c} d^{-}$ & & - & 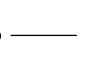 & $\operatorname{dag~kg}{ }^{-1}$ & $\mathrm{mg} \mathrm{L}^{-1}$ \\
\hline $0-3$ & $590^{(1)}$ & 110 & 300 & $2,54^{(1)}$ & 5,5 & $2,50^{(1)}$ & 2,55 & 5,90 & 37,31 & 10,01 & 3,03 & 8,97 \\
\hline $10-13$ & 600 & 130 & 270 & 2,54 & 5,5 & 2,50 & 2,55 & 5,90 & 37,31 & 10,01 & 3,03 & 8,97 \\
\hline $25-28$ & 590 & 90 & 320 & 2,54 & 5,4 & 1,60 & 1,86 & 5,48 & 28,15 & 18,48 & 2,86 & 8,84 \\
\hline
\end{tabular}

(1) Média de três repetições.

manejos realizados com controle mecânico ou com plantas na estrutura do LVA. Nesse manejo, em julho de 2009, foram coletadas, aleatoriamente no centro das entrelinhas, 60 amostras com estrutura indeformada (20 amostras x 3 profundidades), utilizando um amostrador de Uhland com anel volumétrico de $6,40 \mathrm{~cm}$ de diâmetro interno por $2,54 \mathrm{~cm}$ de altura.

Todos os equipamentos utilizados no manejo de controle mecânico das plantas invasoras foram tracionados por um trator Massey Ferguson 275 (massa aproximada de $3.080 \mathrm{~kg}$ ). As operações de controle mecânico das plantas invasoras foram efetuadas sempre que se observavam $90 \%$ da área coberta pelas plantas invasoras e, ou, estas apresentavam cerca de 0,45 m de altura (Alcântara \& Ferreira, 2000; Araujo Junior et al., 2008; Alcântara et al., 2009). Desde a implantação da lavoura, os manejos utilizados nas entrelinhas foram os mesmos, e o número médio de operações adotadas para controle satisfatório das plantas invasoras, durante os anos, variou de acordo com o sistema de manejo adotado.

Nos manejos de plantas invasoras realizados por meio do controle mecânico observou-se o seguinte número de operações: grade (GD): com 16 discos dispostos em V e massa aproximada de $262 \mathrm{~kg}$, trabalhando a $15 \mathrm{~cm}$ de profundidade, em média, foram realizadas três operações anuais; herbicida de pós-emergência (HPós): glyphosate alternadamente com glyphosate $+2,4$-D, com média de três aplicações ao ano; herbicida de pré-emergência (HPré): ametryn + simazine, com média de duas aplicações ao ano; roçadora (RÇ): marca Kamaq KDD 230 ECO Cruiser, de levante hidráulico e acionado pela tomada de força do trator, de massa aproximada de $560 \mathrm{~kg}$, com média de cinco operações anuais; e trincha (TC): marca Tritton 1.300 RB com seis fileiras de martelos de aço fundido e temperado, com massa aproximada de $570 \mathrm{~kg}$, de levante hidráulico e acionado pela tomada de força do trator, com média de cinco operações anuais. Foram coletadas em janeiro de 2010, nas linhas de tráfego das entrelinhas, 180 amostras com estrutura indeformada (5 manejos x 3 profundidades x 12 amostras de solo com estrutura indeformada), para quantificar as alterações que esses manejos possam ter promovido na estrutura do LVA.
Nos manejos de plantas invasoras realizados com o uso de plantas de cobertura e, portanto, sem tráfego de máquinas - amendoim forrageiro (Arachis pintoi) (AF), braquiária (Brachiaria decumbens) (BD), capina manual $(\mathrm{CM})$ realizada com auxílio de enxada (em média, cinco operações anuais), crotalária (Crotalaria juncea) (CR) e soja (Glycine max L.) (SJ) - foram coletadas em janeiro de 2010, no centro das entrelinhas, 180 amostras com estrutura indeformada (5 manejos $\mathrm{x} 3$ profundidades $\mathrm{x} 12$ amostras de solo com estrutura indeformada), para quantificar as alterações que esses manejos possam ter promovido na estrutura do LVA.

As profundidades de amostragem foram de 0-3, 10-13 e 25-28 cm, em razão de a maior influência dos sistemas de manejo de plantas invasoras no solo ocorrer até $30 \mathrm{~cm}$ (Alcântara \& Ferreira, 2000; AraujoJunior et al., 2008).

Para determinação dos Modelos de Capacidade de Suporte de Carga (MCSC) para o manejo de plantas invasoras SC, as amostras com estrutura indeformada com diferentes umidades volumétricas $(\theta)(0,07$ a $0,46 \mathrm{~cm} \mathrm{~cm}^{-3}$ ) foram submetidas ao ensaio de compressão uniaxial, utilizando-se um consolidômetro da marca Board Longyer. As pressões aplicadas às amostras foram de 25, 50, 100, 200, 400, 800 e $1.600 \mathrm{kPa}$. Cada pressão foi aplicada até alcançar 90 \% da deformação máxima (Taylor, 1948; Holtz \& Kovacs, 1981; Bowles, 1986), obtendo-se as curvas de compressão do solo, nas quais foram determinadas as pressões de pré-consolidação $\left(\sigma_{\mathrm{p}}\right)$, de acordo com Dias Junior \& Pierce (1995). Os valores de $\sigma_{\mathrm{p}}$ com os valores de $\theta$ foram ajustados a uma regressão exponencial decrescente do tipo $\sigma_{\mathrm{p}}=10^{(\mathrm{a}+\mathrm{b} \theta)}$ (Dias Junior, 1994), em que $\sigma_{\mathrm{p}}$ é a pressão de pré-consolidação; $\theta$, a umidade volumétrica do solo; e "a" e "b", os coeficientes de ajuste da regressão.

Os modelos de capacidade de suporte de carga das diferentes profundidades para o manejo SC foram comparados utilizando o procedimento descrito em Snedecor \& Cochran (1989).

Para quantificar as alterações que os manejos de plantas invasoras possam ter promovido na estrutura do LVA, as amostras de solo com estrutura indeformada coletadas nos manejos GD, HPós, HPré, RÇ, TC, AF, BD, CM, CR e SJ e com a umidade volumétrica de campo foram submetidas ao ensaio de 
compressão uniaxial, conforme descrito anteriormente, determinando-se a pressão de pré-consolidação, de acordo com Dias Junior \& Pierce (1995).

A seguir, as pressões de pré-consolidação e as umidades volumétricas de campo foram representadas nos MCSC do manejo de plantas invasoras SC e classificadas de acordo com os critérios propostos por Dias Junior et al. (2005). Para obter esses critérios, os MCSC são divididos em três regiões: uma situada acima do limite de confiança superior, considerada como a região na qual a compactação do solo já ocorreu; outra situada entre os limites de confiança, considerada como uma região na qual não há compactação, mas existe tendência de ocorrer; e uma situada abaixo do limite de confiança inferior, considerada como uma região na qual não há compactação do solo (Dias Junior et al., 2005) (Figuras 2 e 3). Para obter a percentagem das amostras que pertencem a uma dessas regiões, o número de amostras pertencentes a uma dessas regiões foi dividido pelo número total de amostras coletadas.

\section{RESULTADOS E DISCUSSÃO}

Os MCSC do LVA estão apresentados na figura 1. Os valores dos parâmetros estimados "a" e "b" do modelo de capacidade de suporte de carga $\left[\sigma_{p}=10^{(a+b \theta)}\right]$, nas profundidades de 0-3, 10-13 e 25-28 cm, variaram de 2,69 a 2,77 e de $-0,99$ a $-1,31$, respectivamente (Figura 1). Os coeficientes de determinação $\left(\mathrm{R}^{2}\right)$ variaram de 0,93 a 0,94 , sendo todos significativos a $1 \%$ (Figura 1).

Os MCSC para as profundidades de $0-3$ e 10-13 cm não foram estatisticamente diferentes (Quadro 2). Assim, uma nova equação foi ajustada, considerando todos os valores de $\sigma_{\mathrm{p}}$ e $\theta$, obtendo-se desse modo um único modelo de capacidade de suporte de carga para as profundidades de 0-3 e 10-13 cm.

O MCSC das profundidades de 0-3 e 10-13 cm e o MCSC da profundidade de $25-28 \mathrm{~cm}$ foram não homogêneos; em razão disso, essas profundidades apresentaram capacidades de suporte de carga diferentes (Quadro 2).
Para analisar os efeitos das operações de manejos de plantas invasoras sobre a estrutura do solo, utilizaram-se os critérios apresentados nas figuras 2 e 3 .

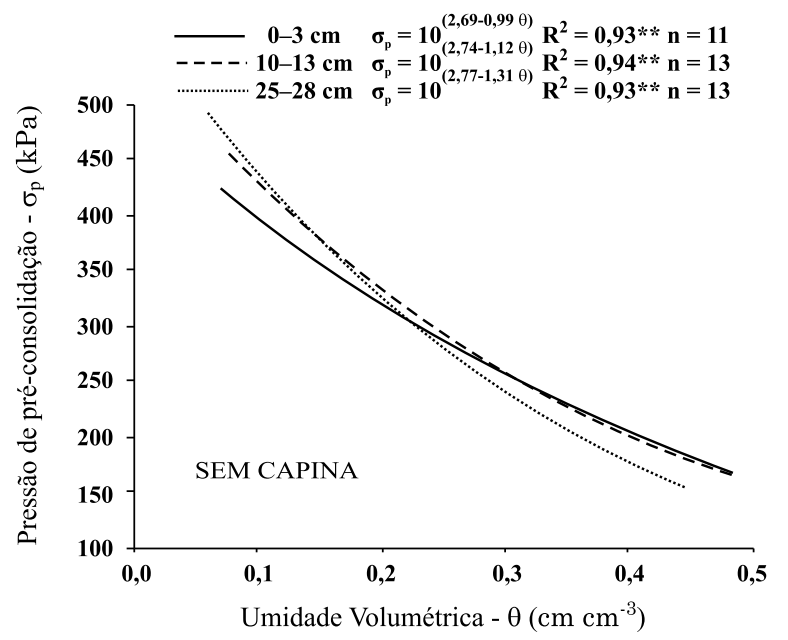

Figura 1. Modelos da capacidade de suporte de carga do Latossolo Vermelho-Amarelo das profundidades de 0-3, 10-13 e 25-28 cm.

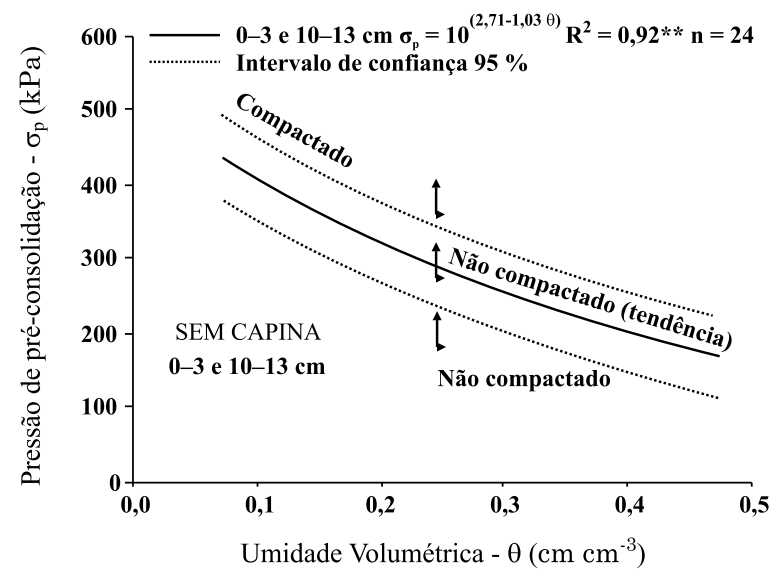

Figura 2. Modelo de capacidade de suporte de carga do Latossolo Vermelho-Amarelo das profundidades de 0-3 e 10-13 cm, com os critérios sugeridos por Dias Junior et al. (2005), para quantificar a compactação causada pelos manejos de plantas invasoras sobre a estrutura do LVA.

Quadro 2. Comparação dos modelos de capacidade de suporte de carga $\left[\sigma_{\mathrm{p}}=10^{(\mathrm{a}=\mathrm{b} \theta)}\right.$, nas profundidades de 0-3, 0-13 e 25-28 cm, para o Latossolo Vermelho-Amarelo, de acordo com o procedimento descrito em Snedecor \& Cochran (1989)

\begin{tabular}{cllll}
\hline \multirow{2}{*}{ Classe de solo } & Profundidade & F & Coeficiente angular, b & Coeficiente linear, a \\
\hline LVA & $0-3 \times 10-13 \mathrm{~cm}$ & $\mathrm{H}$ & $\mathrm{ns}$ & $\mathrm{ns}$ \\
LVA & $0-3$ e $10-13 \times 25-28 \mathrm{~cm}$ & $\mathrm{NH}$ & $* *$ & $\mathrm{~ns}$ \\
\hline
\end{tabular}

F: teste de significância de acordo com Snedecor \& Cochran (1989); H: homogêneo, NH: não homogêneo, ns: não significativo; ** : significativo a $1 \%$. 


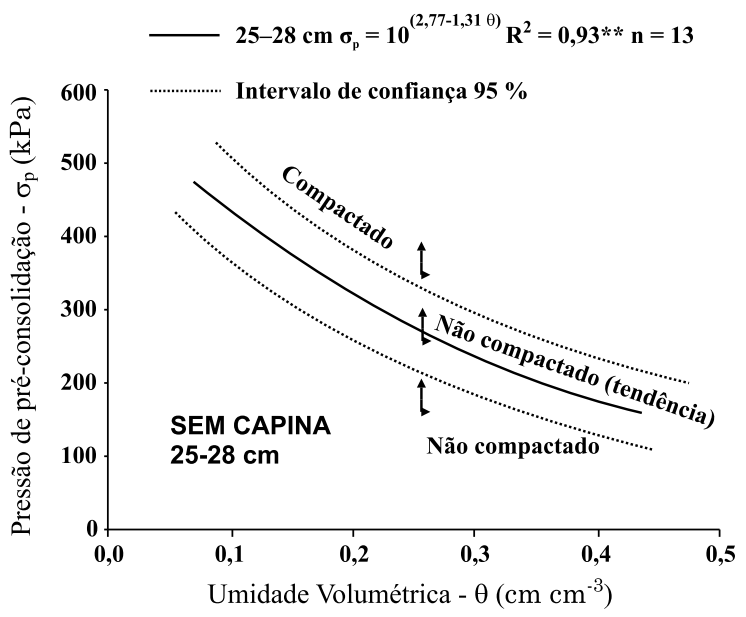

Figura 3. Modelo de capacidade de suporte de carga do Latossolo Vermelho-Amarelo da profundidade de $25-28 \mathrm{~cm}$, com os critérios sugeridos por Dias Junior et al. (2005), para quantificar a compactação causada pelos manejos de planta invasoras sobre a estrutura do LVA.

Na profundidade de 0-3 cm, os manejos de plantas invasoras que mais degradaram a estrutura do LVA foram, em ordem decrescente: GD $>\mathrm{RC}>\mathrm{AF}>\mathrm{TC}$ (Quadro 3).

A gradagem realizada em 2006 promoveu pulverização do solo, com consequente destruição de sua estrutura, o que o tornou mais suscetível à compactação, devido à sua baixa capacidade de suporte de carga. Assim, na profundidade de $0-3 \mathrm{~cm}$, as operações de controle de plantas invasoras realizadas com a GD e com a RÇ nos anos subsequentes promoveram compactação do solo na linha de tráfego do trator em 73 e $58 \%$ das amostras com estrutura indeformada (Quadro 3), respectivamente. Esses resultados corroboram os obtidos por Kurachi \& Silveira (1984), Silveira et al. (1985), Souza \& Melles (1986), Alcântara \& Ferreira (2000), Silva et al. (2006), Araujo Junior (2007), Alcântara et al. (2008) e Araujo Junior (2010).

No manejo de plantas invasoras utilizando AF e $\mathrm{CM}$, na profundidade de $0-3 \mathrm{~cm}$, a compactação do solo pode ter sido causada pelo impacto direto das gotas de chuva e pelos ciclos de secagem e umedecimento, $o$ que promoveu o rearranjamento das partículas sólidas do solo (Araujo Junior et al., 2008), com consequente aparecimento de crostas. Esse processo ficou evidenciado em razão da cobertura não homogênea do solo observada no campo para o $\mathrm{AF}$ e da falta de cobertura do solo logo após a realização da CM.

A compactação do solo promovida pela trincha (TC) na profundidade de $0-3 \mathrm{~cm}$ foi menor do que a promovida pela GD, RÇ e AF. Isso pode ser devido ao fato de a TC ser um implemento que tritura os restos vegetais sem tocar o solo (Fernandes, 2010), dispondo esses resíduos na linha de tráfego do trator, o que auxilia na dissipação das pressões aplicadas por essa operação. Apesar de a RÇ também não tocar o solo, ela tira os resíduos da linha de tráfego do trator, o que, de acordo com Silva et al. (2007), aumenta o risco de compactação em 50 a $70 \%$, quando comparado com o tráfego realizado sobre resíduos.

No sistema de controle de plantas invasoras herbicida de pré-emergência (HPré), a percentagem de amostras compactadas foi de $8 \%$, possivelmente em decorrência do encrostamento superficial. Esse encrostamento pode ter até $5 \mathrm{~cm}$ de espessura a partir da superfície do solo, sendo caracterizado pela quebra dos agregados, promovendo entupimento dos poros, e aumento da resistência mecânica dessa camada, conforme identificado pelos valores das pressões de preconsolidação (Araujo Junior et al., 2008, 2011).

Quadro 3. Percentagem de compactação causada pelos manejos de controle de plantas invasoras sobre a estrutura do LVA nas profundidades de 0-3, 10-13 e 25-28 cm

\begin{tabular}{|c|c|c|c|c|c|c|c|c|c|c|}
\hline & $\mathbf{C M}$ & GD & HPós & $\mathbf{R C ̧}$ & $\mathbf{T C}$ & $\mathrm{AF}$ & BD & $\mathbf{C R}$ & HPré & SJ \\
\hline & \multicolumn{10}{|c|}{$0-3 \mathrm{~cm}$} \\
\hline Compactado (\%) & 8 & 73 & 0 & 58 & 25 & 42 & 0 & 0 & 8 & 0 \\
\hline Não compactado (tendência) (\%) & 75 & 27 & 92 & 42 & 33 & 58 & 58 & 25 & 25 & 42 \\
\hline \multirow[t]{2}{*}{ Não compactado (\%) } & 17 & 0 & 8 & 0 & 42 & 0 & 42 & 75 & 67 & 58 \\
\hline & \multicolumn{10}{|c|}{$10-13 \mathrm{~cm}$} \\
\hline Compactado (\%) & 58 & 73 & 67 & 83 & 75 & 0 & 0 & 0 & 0 & 0 \\
\hline Não compactado (tendência) (\%) & 42 & 9 & 25 & 0 & 25 & 100 & 33 & 33 & 17 & 17 \\
\hline \multirow[t]{2}{*}{ Não compactado (\%) } & 0 & 18 & 8 & 17 & 0 & 0 & 67 & 67 & 83 & 83 \\
\hline & \multicolumn{10}{|c|}{$25-28 \mathrm{~cm}$} \\
\hline Compactado (\%) & 34 & 36 & 83 & 67 & 33 & 0 & 0 & 0 & 8 & 0 \\
\hline Não compactado (tendência) (\%) & 58 & 36 & 17 & 25 & 25 & 92 & 17 & 50 & 0 & 75 \\
\hline Não compactado (\%) & 8 & 28 & 0 & 8 & 42 & 8 & 83 & 50 & 92 & 25 \\
\hline
\end{tabular}

CM: capina manual; GD: grade de discos; HPós: herbicida de pós-emergência; RÇ: roçadora; TC: trincha; AF: amendoim forrageiro; BD =braquiária; CR: crotalária; HPré: herbicida de pré-emergência; SJ: soja. 
Por outro lado, os manejos de plantas invasoras que mais preservaram a estrutura do LVA, na profundidade de $0-3 \mathrm{~cm}$, foram os sistemas que utilizaram plantas de cobertura (BD, CR, SJ e HPós), corroborando resultados de Araujo Junior et al. (2008), que mostram o efeito benéfico do sistema radicular na recuperação da estrutura, além da proteção do solo contra processos erosivos, conservando a umidade do solo e contribuindo para a sustentabilidade do sistema.

Na profundidade de $10-13 \mathrm{~cm}$, os manejos de plantas invasoras que mais degradaram a estrutura do LVA foram, em ordem decrescente: RÇ $>$ TC $>$ GD $>$ HPós > CM (Quadro 3).

Para os manejos de plantas invasoras realizados com a GD, RÇ, TC e HPós, na profundidade de 10 $13 \mathrm{~cm}$, a compactação foi resultado da intensidade de tráfego do trator para a realização das operações de controle das plantas invasoras ao longo do ano (Kurachi \& Silveira, 1984; Alcântara \& Ferreira, 2000; Araújo Junior, 2007; Alcântara et al., 2008; Araujo Junior, 2010; Fernandes, 2010). Além disso, a maioria dessas operações foi realizada na estação chuvosa, quando o solo apresentava-se com elevada umidade, estando, portanto, inadequado para o tráfego de máquinas (Silva et al., 2006). Observa-se, nesses manejos, que eles promoveram maior compactação na profundidade de $10-13 \mathrm{~cm}$ do que na de $0-3 \mathrm{~cm}$, corroborando resultados de Silva et al. (2006), Santos et al. (2010) e Araujo Junior et al. (2011). A maior compactação promovida pela GD pode ser explicada pelo fato de que ela pode ter promovido o aumento da compactação abaixo da profundidade de trabalho dos discos (Souza \& Melles, 1986) - nesse caso, realizado a $15 \mathrm{~cm}$ de profundidade. Já a compactação promovida pelos tratamentos RÇ, TC e HPós pode ser justificada pela distribuição das tensões exercidas pelos rodados do trator ao longo do perfil do solo, que pode causar compactação até a profundidade de $16 \mathrm{~cm}$ (Araujo Junior et al., 2011).

$\mathrm{Na} \mathrm{CM}$, a compactação observada na profundidade de 10-13 cm pode estar associada à ação da gradagem pesada realizada a $15 \mathrm{~cm}$ de profundidade em 2006 , antes da implantação da cultura cafeeira. Ressaltase que após essa gradagem não ocorreu tráfego de máquinas nessa área.

Tanto na profundidade de $0-3 \mathrm{~cm}$ quanto na de $10-13 \mathrm{~cm}$, foi observado que os manejos de plantas invasoras que mais preservaram a estrutura do LVA foram os sistemas que utilizaram plantas de coberturas (BD, CR e SJ), uma vez que a presença do sistema radicular auxilia no alívio da resistência mecânica do solo, devido possivelmente à formação de "bioporos" (Foloni et al., 2006), o que foi evidenciado pelos valores de pressão de pré-consolidação iguais a zero na região onde a compactação do solo já havia ocorrido. Segundo Ehlers et al. (1983), os bioporos possibilitam o crescimento das raízes em solos mais compactados, promovendo alívio da resistência mecânica do solo. Nessa profundidade, o manejo realizado com HPré também preservou a estrutura do LVA, sendo isso justificado pelo fato de que o encrostamento promovido por esse sistema de manejo de plantas invasoras só ocorreu na superfície do solo, corroborando os resultados obtidos por Faria et al. (1998), Bertoni \& Lombardi Neto (1999), Alcântara $\&$ Ferreira (2000) e Araujo Junior et al. (2008).

$\mathrm{Na}$ profundidade de $25-28 \mathrm{~cm}$ (Quadro 3), os manejos de plantas invasoras que promoveram maior compactação do LVA foram, em ordem decrescente: HPós > RÇ > GD > CM > TC (Tabela 4). A compactação observada nesses sistemas nessa profundidade pode ser resultado da operação de gradagem pesada feita antes da implantação do experimento e devido à intensidade de tráfego ao longo do ano (Kurachi \& Silveira, 1984; Alcântara \& Ferreira, 2000; Araújo-Junior, 2007; Alcântara et al., 2008; Araujo Junior, 2010; Fernandes, 2010), principalmente quando esses controles eram feitos na estação chuvosa, com o solo apresentando umidade inadequada para o tráfego (Silva et al., 2006).

\section{CONCLUSÕES}

1. Os modelos de capacidade de suporte de carga do Latossolo Vermelho-Amarelo são expressos pelas seguintes equações: $\sigma_{p}=10^{(2,71-1,03 \theta)}$ (para as profundidades de $0-3$ e $10-13 \mathrm{~cm})$ e $\sigma_{\mathrm{p}}=10^{(2,77-1,31}$ e) $($ para a profundidade de $25-28 \mathrm{~cm}$ ).

2. Os manejos de plantas invasoras que promoveram maior compactação do LVA, nas três profundidades, foram grade de discos, roçadora e trincha.

3 . Os manejos de plantas invasoras que promoveram menor compactação do LVA, nas três profundidades, foram braquiária, crotalária e soja.

\section{LITERATURA CITADA}

ASSOCIAÇÃO BRASILEIRA DA INDÚSTRIA DE CAFÉ ABIC. Indicadores da indústria de café no Brasil - 2009. Disponível em: <http://www.abic.com.br/estatisticas $>$. Acesso em 16 out. 2010.

ALCÂNTARA, E.N.; CUNHA, R.L. \& SILVA, R.A. Manejo de mato em cafeeiro: Métodos e coeficientes técnicos utilizados. Inf. Agropec., 29:74-82, 2008.

ALCÂNTARA, E.N. \& FERREIRA, M.M. Efeito de métodos de controle de plantas daninhas na cultura do cafeeiro (Coffea arabica L.) sobre a qualidade física do solo. R. Bras. Ci. Solo, 24:711-721, 2000.

ALCÂNTARA, E.N.; NÓBREGA, J.C.A. \& FERREIRA, M.M. Métodos de controle de plantas daninhas no cafeeiro afetam os atributos químicos do solo. Ci. Rural, 39:749757,2009 
ARAUJO JUNIOR, C.F.; DIAS JUNIOR, M.S.; GUIMARÃES, P.T.C. \& ALCÂNTARA, E.N. Capacidade de suporte de carga e umidade crítica de um Latossolo induzida por diferentes manejos. R. Bras. Ci. Solo, 35:115-131, 2011.

ARAUJO JUNIOR, C.F.; DIAS JUNIOR, M.S.; GUIMARÃES, P.T.C. \& PIRES, B.S. Resistência à compactação de um Latossolo cultivado com cafeeiro, sob diferentes sistemas de manejo de Plantas invasoras. R. Bras. Ci. Solo, 32:2332,2008 .

ARAUJO JUNIOR, C.F. Capacidade de suporte de carga de um Latossolo após três décadas de diferentes manejos de plantas invasoras em uma lavoura cafeeira. Lavras, Universidade Federal de Lavras, 2010. 143p. (Tese de Doutorado)

ARAUJO JUNIOR, C.F. Modelos de capacidade de suporte de carga de um Latossolo submetido a diferentes sistemas de manejo de plantas daninhas em lavoura cafeeira. Lavras, Universidade Federal de Lavras, 2007. 132p. (Tese de Mestrado)

BLANCO, H.G.; OLIVEIRA, P.A. \& PUPO, E.I.H. Efeitos da época de controle do mato sobre a produção de uma lavoura de café em formação. In: CONGRESSO BRASILEIRO DE PESQUISAS CAFEEIRAS, 6., Ribeirão Preto, 1978. Anais. Rio de Janeiro, IBC/GERCA, 1978. p.56-57.

BOWLES, J.E. Engineering properties of soils and their measurements. 3.ed. New York, McGraw Hill, 1986. 218p.

BRASIL. Ministério da Agricultura e Reforma Agrária. Normais climatológicas 1961-1990. Brasília, 1992. 84p.

CANELLAS, L.P.; SANTOS, G.A. \& AMARAL SOBRINHO, N.M.B. Reações da matéria orgânica. In: SANTOS, G.A. \& CAMARGO, F.A.O., ed. Fundamentos da matéria orgânica do solo: Ecossistemas tropicais e subtropicais. Porto Alegre, Genesis, 1999. p.69-90.

COMPANHIA NACIONAL DE ABASTECIMENTO - CONAB. Acompanhamento da Safra Brasileira Café Safra 2011 segunda estimativa. <http://www.conab.gov.br/acesso>. Acesso em 13 jul. de 2011.

DIAS JUNIOR, M.S. Compactação do Solo. In: NOVAIS, R.F.; ALVAREZ V., H.V. \& SCHAEFER, C.E.G.R. Tópicos em ciência do solo. Viçosa, MG, Sociedade Brasileira de Ciência do Solo, 2000. v.1, p.55-94.

DIAS JUNIOR, M.S. Compression of the soils under longterm tillage and wheel traffic. East Lansing, Michigan State University, 1994. 114p. (Tese de Doutorado)

DIAS JUNIOR, M.S.; LEITE, F.P.; LASMAR JÚNIOR, E. \& ARAUJO JUNIOR, C.F. Traffic effects on the soil preconsolidation pressure due to Eucalyptus harvest operations. Sci. Agric., 62:248-255, 2005.

DIAS JUNIOR, M.S. \& PIERCE, F.J. Revisão de literatura. O processo de compactação do solo e sua modelagem. R. Bras. Ci. Solo, 20:175-182, 1996.

DIAS JUNIOR, M.S. \& PIERCE, F.J. A simple procedure for estimating preconsolidation pressure from soil compression curves. Soil Technol., 8:139-151, 1995.
EHLERS, W.W.; KOPKE, F.; HESSE, F. \& BOHM, W. Penetration resistance and growth root of oats in tilled and untilled loess soil. Soil Till. Res., 3:261-275, 1983.

EMPRESA BRASILEIRA DE PESQUISA AGROPECUÁRIA EMBRAPA. Centro Nacional de Pesquisa de Solos. Sistema brasileiro de classificação de solos. 2.ed. Rio de Janeiro, 2006. 306p.

FERNANDES, A.C.F. Plantas daninhas no cafezal. Disponível em: <https://www.cooxupe.com.br/folha/fevereiro05/ pag9.htm>. Acesso em 07 out. 2010.

FOLONI, J.S.S.; LIMA, S.L. \& BÜLL, L.T. Crescimento aéreo e radicular da soja e de plantas de cobertura em camadas compactadas de solo. R. Bras. Ci. Solo, 30:49-57, 2006.

GYSI, M. Compaction of a Eutric Cambisol under heavy wheel traffic Switzerland: Field data and a critical state soil mechanics model approach. Soil Till. Res., 61:133-142, 2001.

HOLTZ, R.D. \& KOVACS, W.D. An introdution to geotechnical engineering. Englewood Cliffs, Prentice-Hall, 1981. 733p.

KURACHI, S.A.H. \& SILVEIRA, G.M. Compactação do solo em cafezal provocada por diferentes métodos de cultivo. Campinas, Instituto Agronômico de Campinas, 1984. 28p.

LARSON, W.E.; BLAKE， G.R.; ALLMARAS， R.R.; VOORHEES, W.B. \& GUPTA, S.C. Mechanics and related processes in structured agricultural soils. The Netherlands, Kluwer Academic Publishers, 1989. 273p. (NATO Applied Science, 172)

MARINO, L.K. Expansão desordenada da cultura cafeeira. In: Anuário da Agricultura Brasileira. São Paulo, Consultoria \& Comércio, 2002. p.210-214.

MIGUEL, A.E.; PEREIRA, J.E. \& OLIVEIRA, J.A. Mobilização de nutrientes pelas plantas daninhas na cultura do café. In: CONGRESSO BRASILEIRO DE PESQUISAS CAFEEIRAS, 8., Campos do Jordão, 1980. Anais... Rio de Janeiro, IBC/GERCA, 1980. p.44-46.

SANTOS, G.A. Capacidade de suporte de carga de um Latossolo cultivado com cafeeiros sob métodos associados de controle de plantas daninhas. Lavras, Universidade Federal de Lavras, 2006. 103p. (Tese de Doutorado)

SANTOS, G.A.; DIAS JUNIOR, M.S.; GUIMARÃES, P.T.G. \& PAIS, P.S.M. Suscetibilidade à compactação de um Latossolo Vermelho-Amarelo submetido a diferentes métodos de controle de plantas invasoras na cultura cafeeira. Coffee Sci., 5:123-136, 2010.

SANTOS, J.C.F.; MARCHI, G. \& MARCHI, E.C.S. Cobertura do solo no controle de plantas daninhas do café. Planaltina, Embrapa, 2008. 54p. (Documentos, 226)

SILVA, A.R.; DIAS JUNIOR, M.S.; GUIMARAES, P.T.G. \& ARAUJO JUNIOR, C.F. Modelagem da capacidade de suporte de carga e quantificação dos efeitos das operações mecanizadas em um Latossolo Amarelo cultivado com cafeeiros. R. Bras. Ci. Solo, 30:207-216, 2006.

SILVA, A.R.; DIAS JUNIOR, M.S. \& LEITE, F.P. Camada de resíduos florestais e pressão de pré-consolidação de dois Latossolos. Pesq. Agropec. Bras., 42:89-93, 2007. 
SILVA, R.H. \& ROSOLEM, C.A. Crescimento radicular de espécies utilizadas como cobertura decorrente da compactação do solo. R. Bras. Ci. Solo, 26:253-260, 2001.

SILVEIRA, G.M.; KURACHI, S.A. \& FUJIWARA, M. Métodos mecânicos e químicos de controle de ervas daninhas em cafezal. Bragantia, 44:173-178, 1985.

SNEDECOR, G.W. \& COCHRAN, W.G. Statical methods. 8.ed. Ames, Iowa State University Press, 1989. 503p.

SOUZA, I.F. \& MELLES, C.C.A. Controle de plantas daninhas. In: SIMPÓSIO SOBRE FATORES QUE AFETAM A PRODUTIVIDADE DO CAFEEIRO, 1., Poços de Caldas, 1984. Anais... Piracicaba, Potafos, 1986. p.401-408.
TAYLOR, D.W. Fundamentals of soil mechanics. New York, J. Wiley, 1948. 770p.

WILDNER, L.P. Utilização de espécies de verão para adubação verde, cobertura e recuperação do solo em Santa Catarina. In: ENCONTRO NACIONAL DE ROTAÇÃO DE CULTURAS, 2., Campo Mourão, 1992. Anais... Campo Mourão, AEACM, 1992. p.144-160. 
Paula Sant'Anna Moreira Pais et al.

R. Bras. Ci. Solo, 35:1949-1957, 2011 